\title{
Cambios en la condición física en estudiantes que ingresan al programa de Cultura Física, Deporte y Recreación, cohorte 2013-I*
}

\author{
Laura Elizabeth Castro-Jiménez ${ }^{* *}$ \\ Yenny Paola Argüello-Gutiérrez ${ }^{* * *}$ \\ Nicole Vásquez ${ }^{* * * *}$ \\ José Valderrama ${ }^{* * * * *}$ \\ Heider Tovar ${ }^{* * * * * *}$ \\ Oskar Godoy ${ }^{* * * * *}$ \\ Daiver Sabogal $\left.\right|^{* * * * * * *}$
}

Recibido: diciembre 11 de 2017 • Aceptado: mayo 7 de 2018

\begin{abstract}
"Artículo de investigación, asociado al proyecto de investigación Cambios en la condición física en estudiantes que ingresan al programa de cultura física, deporte y recreación desde el 2013-II al 2016-II, financiado por la Universidad Santo Tomás con el grupo de investigación Cuerpo, sujeto y educación. Citar como: Castro, L., Arguello, Y., Vásquez, N., Valderrama, J., Tovar, H., Godoy, O. y Sabogal, D. (2018). Cambios en la condición física en estudiantes que ingresan al programa de Cultura Física, Deporte y Recreación, cohorte 2013-I. Cuerpo, Cultura y Movimiento, 8(1), 27-45. DOI: https://doi.org/10.15332/2422474X.5120
\end{abstract}

" Docente de la Facultad de Cultura Física, Deporte y Recreación. Universidad Santo Tomás, Bogotá D. C., Colombia. Grupo de investigación Cuerpo, sujeto y educación. Correo electrónico: laura.castro@usantotomas.edu.co / ORCID: https://orcid.org/0000-0001-5166-8084

*** Docente de la Facultad de Cultura Física, Deporte y Recreación. Universidad Santo Tomás, Bogotá, Colombia. Grupo de investigación Cuerpo, sujeto y educación. Correo electrónico: yenny.arguello@iberoamericana.edu.co / ORCID: https://orcid.org/0000-0001-8335-4936

"***** Estudiante de la Facultad de Cultura Física, Deporte y Recreación. Universidad Santo Tomás, Bogotá, Colombia. Grupo de investigación Cuerpo, sujeto y educación. Correo electrónico: nicolevasquez@usantotomas.edu.co

"***** Estudiante de la Facultad de Cultura Física, Deporte y Recreación. Universidad Santo Tomás, Bogotá, Colombia. Grupo de investigación Cuerpo, sujeto y educación. Correo electrónico: josevalderrama@usantotomas.edu.co

******* Estudiante de la Facultad de Cultura Física, Deporte y Recreación. Universidad Santo Tomás, Bogotá, Colombia. Grupo de investigación Cuerpo, sujeto y educación. Correo electrónico: heidertovar@usantotomas.edu.co 


\section{Resumen}

El propósito de este escrito fue determinar los cambios existentes en la condición física, en la cohorte de estudiantes de Cultura Física, Deporte y Recreación del 2013-I al 2017-I. El estudio fue de corte cuantitativo, con una cohorte de seguimiento prospectivo desde el 2013-I y hasta el 2017-I, con alcance descriptivo. Los criterios de inclusión fueron, estudiantes de la Facultad, quienes hubieran permanecido activos desde el ingreso y hasta la fecha del corte. Los componentes que se evaluaron fueron: la fuerza (a través de dinamometría), la flexibilidad (test de Wells) y la agilidad (prueba de obstáculos y adaptación al balón). Se inició el seguimiento a 153 estudiantes desde el 2013-I y hasta el 2017-I, de los cuales solo terminaron 38. Se encontró una disminución de los valores de fin de carrera de las pruebas de flexibilidad (media pre $8.51 \mathrm{y}$ pos 3), prueba de obstáculos (media pre 31,35 segundos y pos 43,61 segundos). Las pruebas de fuerza de mano y adaptación al balón mejoraron su media, pero sus rangos disminuyeron con respecto a la prueba de inicio. Finalmente, mejoraron los valores de la fuerza de espalda.

Palabras clave: aptitud física, criterios de admisión escolar, estudiantes.

Estudiante de la Facultad de Cultura Física, Deporte y Recreación. Universidad Santo Tomás, Bogotá, Colombia. Grupo de investigación Cuerpo, Sujeto y Educación. Correo electrónico: oskargodoy@usantotomas.edu.co

Estudiante de la Facultad de Cultura Física, Deporte y Recreación. Universidad Santo Tomás, Bogotá, Colombia. Grupo de investigación Cuerpo, Sujeto y Educación. Correo electrónico: daiversabogal@usantotomas.edu.co 


\title{
Changes in physical condition in students entering the program of Physical Culture, Sports and Recreation, Cohorte 2013-I
}

\begin{abstract}
The purpose of this paper was to determine the changes in physical condition in the cohort of students of Physical Culture, Sports and Recreation from 2013-I to 2017-I. The study was quantitative, with a prospective follow-up cohort from 2013-I until 2017-I, with a descriptive scope. The inclusion criteria were students of the Faculty, who had remained active from the time of admission and until the date of the cut. The components that were evaluated were: strength (through dynamometry), flexibility (Wells test) and agility (obstacle test and adaptation to the ball). The follow-up began to 153 students from 2013-I and up to 2017-I, of which only ended 38. A decrease of the end-of-career values of the flexibility tests was found (average pre 8,51 and post 3), obstacle test (average pre 31,35 seconds and post 43,61 seconds). The tests of hand strength and adaptation to the ball improved their average, but their ranges decreased with respect to the start test. Finally, the values of back strength improved.
\end{abstract}

Keywords: physical fitness, school admission criteria, students. 


\section{Introducción}

Las universidades a nivel mundial que ofertan los programas de Cultura Física y Educación Física, poseen pruebas de admisión que valoran en su gran mayoría las capacidades físicas, condicionales y coordinativas de sus aspirantes. El programa de la Universidad Santo Tomás se ha posicionado en el ámbito académico colombiano como un proyecto innovador, donde ofrece al estudiante la posibilidad de formarse como profesional, desde las competencias del saber, el ser y el hacer; aspectos que posibilitan encaminarse hacia potencialidades profesionales, afrontando los retos que el sector exige de quienes ocupan estas áreas.

La Facultad de Cultura Física, Deporte y Recreación de la Universidad Santo Tomás posee un proceso de admisiones, el cual evalúa la competencia cinética corporal, las competencias básicas y el contexto sociocultural de los jóvenes; en donde las personas que tengan interés en cursar este programa, deben inclinarse por la práctica de la actividad física y el deporte, mostrando interés por los hábitos de vida saludable y la formación integral del individuo a través de la praxis motora; evidenciando a su vez, un nivel de acondicionamiento físico, competencias básicas y niveles de desarrollo personal que le posibiliten la adquisición de los conocimientos durante el curso de la carrera (Córdoba, 2013). La importancia de estas evaluaciones es determinar un criterio básico de selección, así como de reconocimiento del perfil de quien ingresa al programa y del desarrollo de sus habilidades y conocimientos. De esta manera, el título de grado en Cultura Física, Deporte y Recreación prepara al estudiante para trabajar en diferentes orientaciones de la actividad física y deportiva, tales como el deporte, la actividad física y de salud, la educación física, el ocio y recreación deportivas y la gestión deportiva (Córdoba, 2013).

La primera convocatoria tuvo dos momentos, el primero, se hacía en las instalaciones del Campus San Alberto Magno, más específicamente un circuito en la pista atlética, para lo cual se conducían los estudiantes en buses a la sede del campus desde la sede central; y un segundo momento, que correspondía a las entrevistas, las cuales se realizaban en la sede central de la Universidad. En esta primera admisión se presentaron quince estudiantes, de los cuales fueron admitidos doce. Posteriormente en el año 1999, se incluyó la prueba escrita, que consistía en una evaluación en la que en cada 
campo (deportivo, pedagógico, recreación, salud, investigación y estético) se diseñaron preguntas que indicaran los conocimientos mínimos que el aspirante debería tener para su ingreso. Al final de cada proceso de ingreso se hacía una realimentación con el Departamento de Admisiones y allí se evaluaban los criterios en las diferentes dimensiones. A partir del 2005, se realizaron pruebas de ingreso, las cuales medían las capacidades condicionales, coordinativas, habilidades básicas terrestres y acuáticas. Sin embargo, en el 2008, estas pruebas fueron suspendidas, debido a que no se encontraba relación con los espacios académicos ofertados dentro del plan de estudio del programa, siendo así, la entrevista, en ese momento, el único medio por el cual se identificaba el perfil de ingreso al programa. Debido al cambio de decanatura en el 2011, la acreditación en alta calidad del programa, el ajuste curricular y la necesidad de identificar el perfil de ingreso de los aspirantes, se creó el "Modelo de ingreso al programa de pregrado en Cultura Física, Deporte y Recreación”, el cual tuvo su etapa de diseño, pilotaje y validación durante el 2011 y parte del 2012, dentro del cual, se evaluaron las competencias cinético-corporales, y competencias básicas (prueba escrita y entrevista) y contexto sociocultural de los aspirantes, como se mencionó anteriormente. Dentro de las competencias cinético-corporales, se midieron capacidades físicas, como lo son el consumo máximo de oxígeno, flexibilidad, fuerza (mano, espalda, miembros inferiores) y velocidad, habilidades en tierra, agilidad y adaptación al móvil, y habilidades en agua (Córdoba, 2013).

Al hacer una verificación de las investigaciones internacionales en la literatura científica sobre los procesos de admisión y las condiciones de egreso con relación a la condición física, se encontró una investigación de Valdés, Godoy, Herrera y Durán en el 2015, quienes refieren que los estudiantes universitarios al encontrarse en una etapa de constantes cambios como lo pueden ser las largas jornadas de estudios, clases en diversos horarios, aumento de actividades en horas de la noche, o escasez de presupuesto, por nombrar algunas de las causas para tomar hábitos de vida inadecuados durante su formación en la universidad. No obstante, estos autores mencionan que la relación con respecto al ejercicio y los hábitos alimenticios en la carrera, se manifiesta de manera poco saludable, pues estos estudiantes, aunque sus temáticas a lo largo de sus estudios incluyan mejora de calidad de vida, en general, por los resultados encontrados, tienden a tener un bajo 
consumo de frutas, verduras y alimentos integrales; en cambio, se presenta una elevada ingesta de bebidas con azúcar, comida chatarra, galletas y snack, entre otros (Valdés, Godoy, Herrera y Durán, 2015). Estos mismos autores referencian que la condición física en los estudiantes de carreras relacionadas con la actividad física alcanza mejor rendimiento en comparación a estudiantes de otras carreras y lo relacionan con el mayor gasto energético y no por que se alimenten de forma correcta.

Por su parte, Arboleda (2014) da cuenta de un estudio relacionado con la condición física y la salud en estudiantes universitarios al iniciar y terminar sus carreras; en el cual, en primera instancia, hace la aclaración existente entre estas dos variables y la actividad física, y todo lo que puede desbordar el sedentarismo de las mismas; por otro lado, menciona los parámetros que para él son pertinentes en la condición física, como talla, peso, IMC, adiposidad, flexibilidad isquiosural, potencia de miembros inferiores y aptitud cardiorrespiratoria y que se deben medir en los estudiantes universitarios que inician y terminan sus carreras. Para esto, el autor realizó una encuesta de la cual se obtenían la historia familiar y personal de enfermedades, siendo la hipertensión (HTA) la más predominante; la cantidad de práctica deportiva arrojó que el resultado no cumple con las recomendaciones mínimas de actividad física para la salud sugeridas; los valores de peso, índice de masa corporal (IMC) y adiposidad en el grupo de finalizar la carrera fueron mayores, aumentando más en mujeres. Los estudiantes de esta investigación pertenecían a carreras relacionadas con la actividad física, quienes debieran promover buenos hábitos desde el inicio y hasta el final de su formación universitaria, dando ejemplo a aquellos que ven desde otro entorno la formación universitaria.

Otra investigación que evalúa las condiciones físicas de los universitarios, fue la de Alonso, Gutiérrez y Pino (2012), quienes evaluaron composición corporal, fuerza muscular, flexibilidad y consumo máximo de oxígeno a 217 estudiantes de la Universidad de Vigo, España, encontrando que, en las variables de agarre manual, el consumo de oxígeno y fuerza explosiva, las mujeres tenían un mayor porcentaje de personas con bajo aptitud física. La situación opuesta ocurrió con la variable de flexibilidad, los hombres mostraron una menor capacidad. Desde una perspectiva global, la fuerza explosiva de miembros inferiores fue la variable que tuvo peor aptitud con $65,3 \%$. Es así, como se ha encontrado en los diferentes estudios que los 
estudiantes que inician vida universitaria tienen mayor riesgo de aminorar sus condiciones de salud por el cambio de estilo de vida, por lo cual es importante reconocer qué pasa con los estudiantes de cultura física quienes son los profesionales futuros que deben promover salud y prevenir enfermedad. Por ello, esta investigación tuvo por objetivo determinar los cambios existentes en la condición física, en la cohorte de estudiantes de Cultura Física, Deporte y Recreación del 2013-I.

\section{Metodología}

El estudio es de corte cuantitativo, con una cohorte de seguimiento prospectivo desde el 2013-I y hasta el 2017-I. El diseño de la investigación es de alcance descriptivo, en el cual se busca conocer los cambios en la condición física desde el ingreso a la universidad hasta el corte en el 2017-I, de los estudiantes de Cultura Física, Deporte y Recreación de la Universidad Santo Tomás sede Bogotá que ingresaron en el 2013-I.

Criterios de inclusión: hombres y mujeres estudiantes de la Facultad de Cultura Física, Deporte y Recreación de la Universidad Santo Tomás, quienes hayan permanecido activos como estudiantes desde el ingreso y hasta la fecha del corte que es 2017-I.

Criterios de exclusión: personas que presentan lesiones tales como desgarros de la musculatura isquiotibial y los cuádriceps o cualquier otra que les imposibilite desarrollar todas las pruebas físicas; fracturas, cirugías o dolor en la columna vertebral en los últimos 3 meses, que les impida realizar las pruebas pos de condición física, y estudiantes que no hayan permanecido activos de manera constante en el período de seguimiento.

Tipo de muestra: se tomó la población total resultante de la cohorte 2013-I después de aplicar los criterios de exclusión, para evitar sesgo de la información.

\section{Instrumento}

Las pruebas escogidas para su aplicación ya están validadas a nivel internacional (se utilizaron equipos que hacen parte del Laboratorio de Fisiología 
de la Facultad de Cultura Física, Deporte y Recreación) y se especifican en el cuadro a continuación:

Tabla 1. Pruebas de condición física

\begin{tabular}{ccc}
\hline Componente & Pruebas & Validación \\
\hline Fuerza (espalda y mano) & Dinamometría & $\begin{array}{c}\text { Grosser, Starischka y } \\
\text { Zimmermann (1988), } \\
\text { Serrano } \text { et al. (2009), } \\
\text { Miranda (2011). }\end{array}$ \\
& & Correa, Rodríguez, \\
& Camargo y Morales, \\
& Test de Wells & (2016). \\
\hline Flexibilidad & & González De los Reyes, \\
& (2008). \\
\hline Agilidad & Prueba de obstáculos & \\
& Adaptación al balón &
\end{tabular}

Fuente: Córdoba, Modelo de ingreso a la Facultad de Cultura Física, Deporte y Recreación (2013).

La evaluación a través de dinamometría de mano tiene como objetivo medir la fuerza isométrica o potencia muscular de los músculos flexores de mano y antebrazo. Para su evaluación,

[...] el sujeto toma el dinamómetro con su mano de preferencia y aprieta lo más fuerte que pueda, manteniendo el dinamómetro ligeramente alejado del cuerpo, para que no lo toque en ningún momento de la prueba. El sujeto debe procurar mantener la presión de modo progresivo y continuado por lo menos durante dos segundos. Mientras la ejecución no se puede sacudir el aparato, ni cambiar la postura del cuerpo ni la posición del dinamómetro o utilizar algún apoyo. El resultado del test será el mejor obtenido en dos intentos. (Córdoba, 2013, p. 53)

La dinamometría de espalda tiene como objetivo medir la fuerza isométrica en los músculos de la parte posterior del cuerpo. Para su evaluación,

[...] el participante se ubica encima de la plataforma con las rodillas semiflexionadas (ángulo $130^{\circ}-140^{\circ}$ ) y sujetando la barra con una presa dorsal para la mano derecha y palmar para la mano izquierda. Una vez colocado, debe tirar de la barra lo máximo posible hacia arriba, implicando piernas y tronco, sin levantar los pies. La aguja se queda señalando la 
máxima fuerza. La fuerza se realiza entre 5 y 10 segundos, máximo. Durante la ejecución no se puede sacudir el aparato, ni cambiar la postura del cuerpo, ni la posición del dinamómetro o utilizar algún apoyo. (Córdoba, 2013, p. 55)

La flexibilidad se evaluó con el objetivo de valorar de forma global la elasticidad y flexibilidad posterior del tronco y miembros inferiores. Para esto, el sujeto

[...] debe estar sentado frente al cajón, apoyar la planta de los pies en su parte frontal y la punta de los dedos de las manos en el borde de la tapa superior. Mantener las rodillas bien extendidas, flexionar el tronco adelante, intentando llegar lo más lejos posible. Tiene que ir empujando la regla lenta y progresivamente, sin movimientos bruscos, con manos y dedos extendidos. (Córdoba, 2013, p. 49)

Se utilizó un cajón de las siguientes dimensiones: ancho, $45 \mathrm{~cm}$; largo, $35 \mathrm{~cm}$ y alto, $32 \mathrm{~cm}$. La tapa superior de $55 \mathrm{~cm}$ de largo por $45 \mathrm{~cm}$ de ancho, sobrepasando en $15 \mathrm{~cm}$ por la parte donde se apoya.

Con relación a las pruebas de agilidad, se realizó la prueba de obstáculos cuyo objetivo fue evaluar la agilidad de movimiento del ejecutante. Para esto, la prueba consistió en realizar un circuito de cuatro elementos, dos veces seguidas. La relación secuencial de lo que se realiza en esta prueba es la siguiente:

1. Desde la posición de salida (S), a la voz del juez, se pone en marcha el cronómetro para indicar el comienzo de la prueba. 2. Dirigirse hacia los conos situados delante de la colchoneta (C). 3. Desde los dos conos (entre los elementos verticales que marcan y sin derribarlos), realizar un rollo adelante sobre la colchoneta (C), sin derribar la banderola (B). 4. Dirigirse hacia la valla (V1) rodeándola en sentido contrario a las manecillas del reloj y pasándola por debajo. 5. A la salida de la valla dirigirse hacia el cajón sueco $(\mathrm{P})$ para superarlo libremente entre los planos imaginarios de sus dos extremos. 6. Inmediatamente después, dirigirse a la valla (V2) rodeándola en el sentido de las agujas del reloj y pasándola por debajo. 7. Dirigirse nuevamente a la valla (V1) y saltarla. 8. Repetir el circuito dirigiéndose a la colchoneta $(\mathrm{C})$ rodeando el cajón sueco $(\mathrm{P})$. A partir de aquí se repite el orden secuencial antes señalado en los puntos 3, 4, 5, 6 y 
7. 9. Una vez franqueada la valla por segunda vez (V1), dirigirse a la línea de meta $(\mathrm{M})$. El cronómetro se parará cuando se pise detrás de la línea de meta. Para el diseño de la pista se deben tener 6 metros entre ambas vallas, 4 metros entre colchoneta y cajón sueco. $0,85 \mathrm{~m}$ de altura de la valla para hombres y $0,75 \mathrm{~m}$ de altura de la valla para mujeres. $0,86 \mathrm{~m}$ de altura en el cajón sueco para hombres y $0,71 \mathrm{~m}$ de altura en el cajón sueco para mujeres. (Córdoba, 2013).

Y la prueba de adaptación al balón tuvo como objetivo medir la coordinación dinámica general del ejecutante y la coordinación ojo mano, así como el ojo pie. Los materiales que se necesitan para esta prueba son un balón de voleibol, 5 obstáculos de 1.70 metros de altura colocados en línea: el primero situado a una distancia de un metro de la línea de salida y el resto separados dos metros el uno del otro. La distancia entre la línea de salida y el primer obstáculo es de un metro. La distancia entre el quinto obstáculo y la zona de lanzamiento es de un metro. El espacio de la zona de lanzamiento es de un metro de ancho por tres metros de largo, y la distancia de esta a la pared es de 2.5 metros. El ancho del recorrido es de tres metros. Tanto la salida como la llegada se inician entre dos conos situados en la misma línea, a una distancia de tres metros entre sí. En la pared frente a la zona de lanzamiento hay un aro de rítmica de $80 \mathrm{~cm}$ de diámetro, situado en la pared, con el borde inferior a 1,5 metros del suelo; y un rectángulo de 1.5 metros de ancho por un metro de alto, colocado a ras de tierra y debajo del aro. Se parte de la línea de salida (por detrás de ella y sin pisarla) entre las dos marcas sitas en los extremos de la línea de salida y dejando el primer obstáculo a la derecha, botando el balón de voleibol con una mano u otra, indistintamente, en zig zag entre los obstáculos hasta llegar a la zona de lanzamientos. Colocado dentro de la zona de lanzamiento, realizará sucesivos lanzamientos de balón con una mano o ambas hacia el interior del círculo, hasta que acierte dos veces; a continuación, tendrá que realizar golpes sucesivos con el pie (pudiendo parar el balón con la mano para situarlo en la zona de lanzamiento después de cada uno de ellos), hasta acertar dos veces dentro del rectángulo inferior situado debajo del círculo. Después de controlar el balón dentro de la zona se continuará con una conducción, solo con el pie, dejando inicialmente el primer obstáculo a mano derecha y recorriendo en zig-zag el espacio entre los obstáculos hasta que tanto el balón como el aspirante superan la línea de llegada entre los dos conos. 
Las pruebas físicas fueron aplicadas por docentes expertos en el manejo de la toma de datos y se aplicaron a los estudiantes para el ingreso a la Facultad de Cultura Física, Deporte y Recreación, por lo que se tienen ya almacenados los datos preingreso, y se volverán a aplicar en el primer semestre del 2017 datos posingreso. La hipótesis de investigación que guio este estudio es que la condición física de los estudiantes mejora durante su permanencia en la Facultad-(Hi1).

Plan de análisis: para los datos cuantitativos se obtuvieron medidas de tendencia central y de dispersión (media, mediana y desviación estándar), posteriormente se hizo una prueba de normalidad con la prueba de ShapiroWilk, y por último, se realizó un análisis bivariado con una prueba t para muestras relacionadas. La información se almacenó en una base de datos para ser analizada posteriormente a través del programa SPSS versión 21.

\section{Resultados}

Se inició el seguimiento desde el 2013-I a 153 estudiantes y hasta el 2017-I, terminando 38 de ellos. Se retiraron del seguimiento 115 estudiantes, los abandonos se dieron por salida del programa académico, así como aplazamiento de algún semestre.

Los resultados para cada variable se presentaron como media $\mathrm{y} \pm$ desviación estándar (rango). En flexibilidad ( $\mathrm{cm}$ ) se encontró en el 2013-1 que la media era de $8.51 \pm 6.35$ (-4 - 25), en el 2017-1 la media fue de $3 \pm 7.715$ (-14 - 19). En fuerza de mano (kg/f) se encontró en el 2013-1 que la media era de $30.61 \pm 10.23(20-80)$, en el 2017-1 la media fue de $38.22 \pm 4.0(13$ $-57)$.

Con respecto a la fuerza de espalda $(\mathrm{kg} / \mathrm{f})$, en el 2013-1 la media era de $100.13 \pm 29.45$ (49 - 165), en el 2017-1 la media fue de $110.24 \pm 33.67$ (50 - 176). En agilidad (segundos), se encontró en la prueba inicial una media en segundos de $31.35 \pm 5.88$ ( 23 - 46.1), en la prueba del 2017-1 la media en segundos fue de $43.61 \pm 9.47(15.1-68)$. En la prueba de adaptación al balón la prueba inicial dio una media en segundos de $22.81 \pm 4.64$ (14.1 37.7), en la prueba final la media en segundos fue de $21.14 \pm 6.21(15-50)$ (ver Figuras 1 y 2 ). 
Figura 1. Resultados evaluación 2013-1
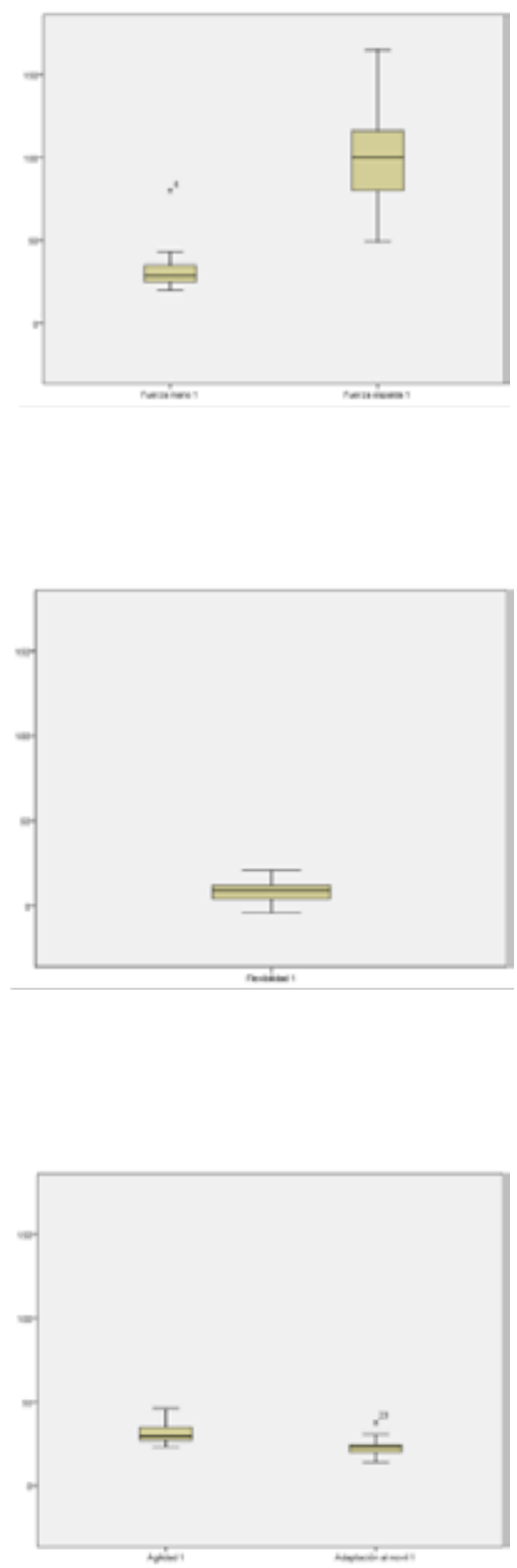

Fuente: elaboración propia. 
Figura 2. Resultados evaluación 2017-1
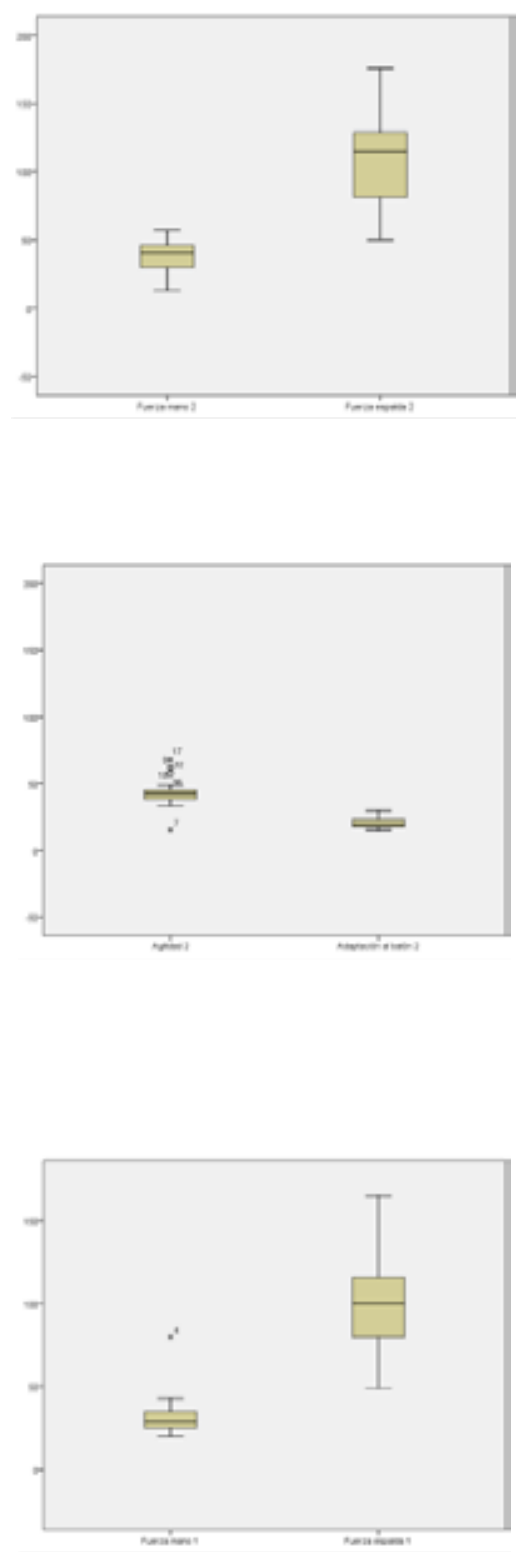

Fuente: elaboración propia. 
En la población se encontró en la variable flexibilidad una disminución del rendimiento global de esta con respecto al ingreso al programa de Cultura Física y al finalizarlo, el coeficiente de correlación fue de 0.617 $(\mathrm{p}=0.000)$. En la variable fuerza de mano se evidenció igualmente una disminución de la fuerza, aunque la media aumentó del antes al después, pero lo que se encuentra es disminución de los valores globales de fuerza así como descenso de la desviación estándar, lo que puede explicar este resultado; el coeficiente de correlación fue de 0.31 ( $\mathrm{p}=0.031$ ). En cambio, los resultados de fuerza de espalda mejoraron con el transcurso del tiempo; el coeficiente de correlación fue de 0.799 ( $\mathrm{p}=0.000)$.

Con relación a los resultados de agilidad, se evidencia aumento del tiempo de ejecución de la prueba, es decir, disminución del rendimiento de la prueba; el coeficiente de correlación fue de 0.210 ( $\mathrm{p}=0.212)$, pero, con los resultados de adaptación al móvil se encontró disminución del tiempo por lo que mejoró su rendimiento. El coeficiente de correlación fue de 0.288 $(\mathrm{p}=0.084)$, aunque los dos no fueron estadísticamente significativos.

\section{Resultados y conclusiones}

El objetivo principal del estudio fue determinar los cambios existentes en la condición física, en la cohorte de estudiantes de Cultura Física, Deporte y Recreación del 2013-I y el resultado más importante fue la disminución de las condiciones físicas en el transcurso de la carrera, como la fuerza prensil, lo cual se ha relacionado con la presencia de riesgo cardiometabólico (Ramírez, Meneses, González y Correa, 2014), también se encontró disminución de los valores de flexibilidad y aumento de los tiempos de respuesta en agilidad, lo que indica que también desmejoraron.

La disminución de las condiciones físicas en esta edad se ha relacionado a un aumento en la presencia de enfermedades crónicas como las de origen cardiometabólico y cardiovascular (Maldonado, Carranza, Ortiz, Gómez y Cortés, 2013; Dadelo y Tamoðauskas, 2005). Así mismo, se ha asociado el beneficio de la actividad física para la salud y el bienestar, pero las características de los entornos universitarios limitan la capacidad de los estudiantes para realizar actividad física o participar en actividades beneficiosas para su salud (Lovasi et al., 2013). 
Frente a la disminución de las cualidades físicas encontradas en la investigación, como por ejemplo la flexibilidad, se ha reportado en el estudio de Arregui y Martínez (2001) que las mujeres tienen mejor flexibilidad que los hombres, como sucedió en las evaluaciones pre y pos, pero ellos repor$\tan$ que los individuos que fueron entrenados tuvieron mejor movilidad que los no entrenados o los entrenados de forma genérica, y en el caso de los estudiantes, a pesar de que están en una carrera en la que realizan actividad física de forma constante, esta cualidad no se mantuvo sino que por el contrario, disminuyó.

Se encontró en otras investigaciones como la de la Universidad Autónoma de Madrid, diferencias significativas de los resultados, donde se evidencia que los resultados de los estudiantes de cultura física están por debajo de los resultados obtenidos por ellos al final del curso; en el caso del test de flexibilidad "Wells", los estudiantes de esta universidad obtuvieron un $26.30 \pm 7.10$ con respecto a los estudiantes de Cultura Física, Deporte y Recreación de la USTA ( $3 \mathrm{~cm} \mathrm{DS} \pm 7.715$ ). Aunque se evaluó con el mismo instrumento, se pueden atribuir las diferencias por el desarrollo de la condición física a lo largo de la carrera, al ser este un pilar importante en el proceso de formación del estudiantado en España, que en el caso colombiano es importante, pero no hace parte de los objetivos de la formación la mejora de las condiciones físicas. Igualmente, en dinamometría se encuentra un aumento de $47.61 \pm 8.72 \mathrm{~kg}$ con respecto a los valores encontrados en los estudiantes de Cultura Física, Deporte y Recreación de la ciudad de Bogotá con un resultado de $38.22 \mathrm{~kg} / \mathrm{f}$ DS \pm 40 (García y Alonso, 2011).

En un estudio que se realizó en 886 estudiantes universitarios colombianos, se encontró que la media de fuerza prensil fue de $32 \mathrm{~kg} / \mathrm{f}$; al comparar estos resultados con los de la población evaluada en esta investigación, se evidencia que la media de fuerza es mayor al tener un resultado de 37 $\mathrm{kg} / \mathrm{f}$. Esta diferencia puede deberse a que son poblaciones que son físicamente activas, pero que, sin embargo, representa un riesgo al ser un valor bajo para la presencia de enfermedad cardiometabólica (Marín, Naclerio, Viejo y Forte, 2007; García-Hermoso et al., 2017).

En relación con la agilidad, no se encontraron investigaciones que evaluaran estas variables en universitarios, pero sí se halló evidencia que indica la importancia de mantenerla en esta edad. Como menciona Nunes (2016), existe la necesidad de diseñar proyectos de educación corporal que lleven a 
la mejora o el mantenimiento de las cualidades físicas del movimiento para evitar complicaciones a futuro.

Las universidades del mundo están recibiendo anualmente una gran cantidad de estudiantes; en Colombia, de acuerdo con el SNIES, en el 2015 se matricularon para el primer curso universitario 785878 estudiantes, los cuales ingresaron a los diferentes programas de pregrado y posgrado en espera de una formación profesional integral (Sistema Nacional de Información de la Educación Superior, 2015). Cuando se piensa en el término de integralidad son muchos los aspectos que entran en juego, las universidades deben velar porque se brinde no solo un desarrollo a nivel cognitivo, sino también en la condición física y en la generación de hábitos saludables. Para ello establecen diferentes estrategias, como por ejemplo, ofertar espacios académicos en los cuales estén presente la actividad física y el deporte para lograr evidenciar la relevancia e importancia que genera hacer actividad física cognitiva y emocionalmente, para poder cumplir con estándares académicos con relación a la condición física de los estudiantes.

Ahora bien, para las universidades que ofertan programas específicos enfocados a la educación física, la cultura física, el deporte y la recreación, está preocupación se torna aún mayor, pues los estudiantes no solo deben poseer una condición física adecuada, sino también deben transmitir y desarrollar estilos de vida saludables en donde se plantea un mejor aprovechamiento de los espacios académicos netamente prácticos para así extraer el mayor provecho de dichas clases y así mejorar de forma autónoma su condición física (León y Zea, 2010).

Los resultados de este estudio evidencian que existen cualidades del movimiento que no se trabajan constantemente en el desarrollo de la carrera, lo que genera una disminución global al finalizarla, ocasionando desventajas a nivel profesional ya que se genera disminución de sus condiciones de salud.

En conclusión, es necesario realizar ajustes que permitan mejorar las cualidades físicas que los estudiantes universitarios de Cultura Física, Deporte y Recreación presentan al finalizar sus estudios y que durante el curso de los mismos se trabaje desde las diferentes cátedras para la mejora de las condiciones de salud de la población estudiantil (Sallis, Calfas, Alcaraz, Gehrman y Johnson, 1999). 


\section{Referencias}

Alonso-Fernández, D., Gutiérrez-Sánchez, Á. y Pino-Juste, M. (2012). Health-related physical condition variables in university students. Journal of Human Sport 6 Exercise, 7(1), 331-340. DOI: https://doi.org/10.4100/jhse.2012.71.11

Arboleda, S. (2014). Indicadores de condición física relacionada con la salud en universitarios que inician y terminan sus carreras. Revista Lúdica Pedagógica, 20 (2), 93-102. DOI: https://doi.org/10.17227/01214128.20ludica93.102

Arregui Eraña, J. A. y Martínez de Haro, V. (2001). Estado actual de las investigaciones sobre la flexibilidad en la adolescencia. Revista Internacional de Medicina y Ciencias de la Actividad Física y el Deporte, 1(2), 127-135. DOI: https://doi.org/10.15366/rimcafd

Córdoba, L. (2013). Modelo de ingreso, Facultad de Cultura Física, Deporte y Recreación. Bogotá D. C., Colombia: Ediciones USTA.

Correa-Mesa, J., Rodríguez-Camacho, D., Camargo-Rojas, D. y Morales, J. (2016). Prevalencia de lesiones en luchadores olímpicos pertenecientes a la Liga de Lucha Olímpica de Bogotá, D. C. Revista de la Facultad de Medicina, 64(3Sup), 99-104. DOI: https://doi.org/10.15446/revfacmed.v64n3sup.50971

Dadelo, S. y Tamoðauskas P. (2005). Investigation and assessment of College students' physical development, Physical fitness, and functional capacity. Acta Academiae Olympiquae Estonia, 13(2), 55-66.

García-Hermoso, A., Carrillo, H., González-Ruíz, K., Vivas, A., Triana-Reina, H., Martínez-Torres, J. y Peterson, M. D. (2017). Fatness mediates the influence of muscular fitness on metabolic syndrome in Colombian collegiate students. PloS one, 12(3), e0173932. DOI: https://doi.org/10.1371/journal.pone.0173932

García-Soidán, J. y Alonso Fernández, D., (2011). Valoración de la condición física saludable en universitarios gallegos. Revista Internacional de Medicina y Ciencias de la Actividad Física y del Deporte. 11(44), 781-790 DOI: https:// doi.org/10.15366/rimcafd

González De los Reyes, Y. (2008). Validez, fiabilidad y especificidad de las pruebas de agilidad. Revista UDCA Actualidad \& Divulgación Científica, 11(2), 3139. Recuperado de: http://ref.scielo.org/qs3w4f

Grosser, M., Starischka, S. y Zimmermann, E. (1988). Principios del entrenamiento deportivo: teoría y práctica en todas las especialidades deportivas. Barcelona, España: Martínez Roca. 
León, A. y Zea, R. (2010). Hábitos y factores de riesgo cardiovascular en estudiantes de primer semestre de la Universidad Santo Tomás. Hallazgos Revista de Investigaciones, 7(13), 181-191. DOI: https://doi.org/10.15332/ s1794-3841.2010.0013.09

Lovasi, G., Schwartz-Soicher, O., Quinn, J., Berger, D., Neckerman, K., Jaslow, R., y Rundle, A. (2013). Neighborhood safety and green space as predictors of obesity among preschool children from low-income families in New York City. Preventive medicine, 57(3), 189-193. DOI: https://doi.org/10.1016/j. ypmed.2013.05.012

Maldonado, J., Carranza, C., Ortiz, M., Gómez, C. y Cortés-Gallegos, N. (2013). Prevalencia de factores de riesgo cardiometabólico en estudiantes universitarios de la región centro-occidente, en la Universidad Michoacana de San Nicolás de Hidalgo, México. Revista Mexicana de Cardiología, 24(2), 76-86. DOI: https://doi.org/10.29351/rmhe.v0i11.136

Marín, P., Naclerio, F., Viejo, D. y Forte, D. (2007). Efectos de diferentes protocolos de entrenamiento de fuerza sobre la fuerza máxima, la velocidad, la saltabilidad y el equilibrio en estudiantes universitarios. Kronos, 6(11-12). DOI: https://doi.org/10.15332/tg.mae.2018.00391

Miranda, M. (2011). Análisis dinamométrico de la mano: valores normativos en la población española (memoria para optar al grado de doctor). Facultad de Medicina, Universidad Complutense de Madrid, España. DOI: https://doi. org/10.23853/bsehm.2017.0304

Nunes, L. (2016). Evaluación de la agilidad en un grupo de niños en edad escolar. Viref Revista de Educación Física, 5(2), 1-7. DOI: https://doi.org/10.1016/j. medcli.2016.09.042

Ramírez-Vélez, R., Meneses-Echávez, J., González-Ruíz, K. y Correa, J. (2014). Fitness muscular y riesgo cardio-metabólico en adultos jóvenes colombianos. Nutrición Hospitalaria, 30(4), 769-775. Recuperado de http:/scielo.isciii.es/ scielo.php?script=sci_arttext\&pid=S0212-16112014001100007\&lng=es\&nr $\mathrm{m}=\mathrm{iso}$

Sallis, J., Calfas, K., Alcaraz, J., Gehrman, C. y Johnson, M. (1999). Potential mediators of change in a physical activity promotion course for university students: Project GRAD. Annals of Behavioral Medicine, 21(2), 149-158.

Serrano, M., Collazos, J., Romero, S., Santurino, M., Armesilla, M., del Cerro, J. y de Espinosa, M. (2009). Dinamometría en niños y jóvenes de entre 6 y 18 años: valores de referencia, asociación con tamaño y composición corporal. Anales de Pediatría, 70(4), 340-348. DOI: https://doi.org/10.1016/j.anpedi.2008.11.025 
Sistema Nacional de Información de la Educación Superior - SNIES. (2015). Población estudiantil: matriculados en primer curso. Recuperado de http:// www.mineducacion.gov.co/sistemasdeinformacion/1735/w3-article-212400. html

Valdés, P., Godoy, A., Herrera, T. y Durán, S. (2015). Comparación en hábitos alimentarios y condición física entre estudiantes de educación física y otras carreras universitarias. Revista Nutrición Hospitalaria, 32(2), 829-836. DOI: https://doi.org/10.3305/nh.2015.32.2.9194. 
